\title{
Pain Physical and Emotional
}

\author{
Andrew Hague* \\ Manufacturers of Medical Equipment, United Kingdom
}

*Corresponding author: Andrew Hague, President, Cellsonic Manufacturers of Medical Equipment United Kingdom.

Received Date: May 17, 2019

Published Date: May 28, 2019

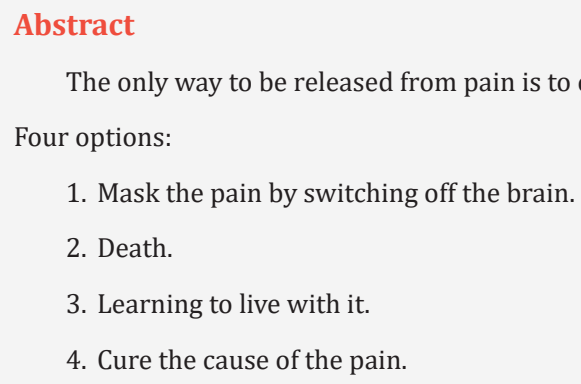

\section{Introduction}

Only the fourth option is acceptable, and this paper will show that it can be done. Unfortunately, the other three options are too common. There are three components in the body that are always found together. If one does not work, the other two cannot work. They are the nerves, veins and arteries. Repairs must suit all three components. Veins and arteries carry blood. If there is no blood at a place in the body, that place cannot be healed. Blood is the life support liquid. In humans even maintaining its temperature is essential.

Nerves carry signals to the brain which is the body's control centre.

Every cell in the body is connected to the brain. The language of the nerve network is pain. From a finger pulling back from a hot surface to the workman stopping to eat, messages flow to the brain and action is taken to keep the body operating. If a message is unable to reach the brain, harm will continue with consequences that can be disastrous. If pain continues and the brain is unable to make a repair, the person suffers. The body's operating system depends on the requests for help being answered and, like an unanswered telephone, will continue ringing until it gets help. It is that perpetual pain that is the subject of the essay.

\section{Masking the Pain}

Drugs can switch off the brain either drastically or slightly. They are never a remedy. Ethanol, a popular poison known as alcohol, has been used by humans since fermentation was discovered by early farmers. Interestingly, reports of animals being allowed to drink alcohol show that the animals also like to be inebriated [1]. If alcohol was originally reserved for celebrations, it eventually became a crutch to carry people through their daily life. Compounding this inadequate answer to a problem is the fact that dependency on the escape or mask becomes addictive.

Worse, whatever the trouble the person wanted to avoid becomes more difficult and they enter a downward spiral (Figure 1). [2] Archaeologists investigating the Neolithic ages, 7,000 years ago, found poppy seeds used medicinally. Before the poppy is ripe, the seed pod can be cut to allow a latex to ooze out and be collected. Observe babies, they put everything in their mouth. Little imagination is required to accept that humans discovered the use of the poppy as an anaesthetic. Poppies are the raw material for opium from which heroin, methane, codeine and thebaine are derived and the synthetic forms of oxycodone, hydrocodone, hydromorphone, and other semisynthetic opiates. 


\section{The Opioid Crisis}

In the American Civil War, the Union Army used 175,000lb $(80,000 \mathrm{~kg})$ of opium tincture and powder and about 500,000 opium pills [1]. During this time of popularity, users called opium "God's Own Medicine". Opium's anaesthetic and addictive powers were well known by the 20th century. Britain had used it to profit from China by forcefully cultivating poppies in India and militarily pushing them on the Chinese [3]. By 1840 there were 10 million Chinese opium addicts; largely due to illegal British imports. Sales were sustained by the users' addiction.

In the late 1990s, around 100 million people or a third of the U.S. population were estimated to be affected by chronic pain [4]. Lower back pain, arthritis, post-surgery pain and cancer were the usual causes of pain and without a cure the patient wanted escape. Pharmaceutical derivatives of opium were the low cost, highly profitable answer to demand. When the pain relievers were launched, they were claimed to be non-addictive. That was soon found to be untrue. "An investigation by the US Senate Committee on Homeland Security and Governmental Affairs detailed the financial ties that exist between opioid manufacturers, advocacy groups, and medical professional societies.

The report exposed patient advocacy groups and professional societies spending millions of dollars to promote messages and policies favoring the interests of the pharmaceutical industry [5]." The patients were addicted to the drugs [6]. Every day, more than 130 people in the United States die after overdosing on opioids. The Centers for Disease Control and Prevention estimates that the total "economic burden" of prescription opioid misuse alone in the United States is $\$ 78.5$ billion a year, including the costs of healthcare, lost productivity, addiction treatment, and criminal justice involvement.

\section{Death}

\section{Breaking bad}

\section{United States, number of opioid deaths By drug*, ${ }^{\prime} 000$}

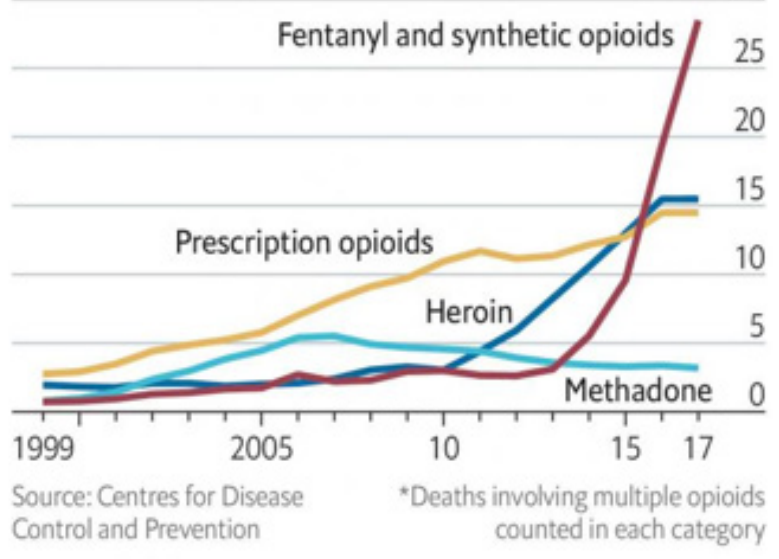

Graph 1: United states number of opioid deaths.
100 million American adults live with chronic pain, many of them with pain so bad it wrecks their work, their families, their mental health and their lives. There are no hard data on how many people with chronic pain die by suicide every year. But there are inferences. The suicide rate among people with chronic pain is known to be roughly twice that for people without chronic pain [7]. Being unable to cure the cause of pain and having a prescriptible anaesthetic for home use available, the doctors put the patients on an addictive drug. When the government saw the folly, they passed laws restricting the amount of opiates (anaesthetics). That left people in pain, doctors in guilt and still no answer [8]. The drugs intended to make life tolerable were doing the opposite. They gave patients a way to kill themselves when they had realised they had two choices: live in pain or die [9]. The doctors were in contact with the patients, not the pharmaceutical companies. The referenced articles are heart breaking (Graph $1 \& 2$ ).

\section{A widening worry \\ Opioid-related deaths per million population}

\section{6 or latest}

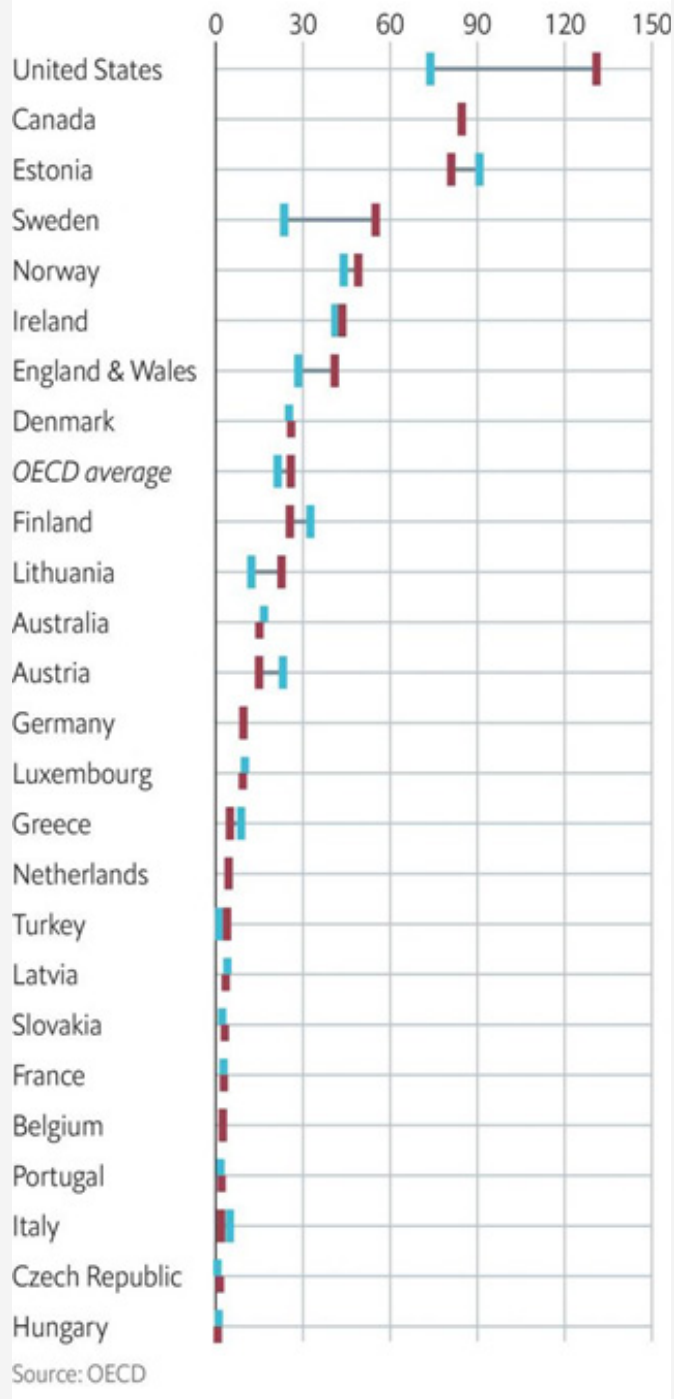

Graph 2: A widening worry. Opioid-related deaths per million population. 


\section{Learn to Live with It}

Is this possible?

\section{Cure the Cause of the Pain}

This is possible and available now. Medicine can be seen in two groups, what can be cured by drugs and what cannot. The pharmaceutical cures include antibiotics and vaccinations. There are also the anaesthetics described above. If the purpose is pain blocking during surgery, then the benefit is clear with the expectation that the pain will pass as the wound heals. What cannot be cured by drugs includes diabetes, cancer and the brain diseases such as Alzheimer's and Parkinson's. Diabetes is more debilitating than painful, so it does not grab the emotive headlines that cancer does. It is the pain from cancer that often alerts the patient. Medical advice is to be checked regularly for cancer and if it can be caught early it can be removed. Maybe it can by selective poisoning of the cancer cells or by targeting with radiation, both methods with side effects. The chemo-radiation cure rate varies from low to dismal.

\section{Cancer}

CellSonic has the only known cure for cancer. I made the discovery almost three years ago. The technology uses a nonsurgical, irreversible electroporation whilst simultaneously applying a short duration, high pressure pulse to the tumour. The explanations, protocols and procedures are already published [10] (Figure 1). When I received the report from the doctor with this photo, I was shocked. We seldom see cancer. It is usually inside. Here a breast tumour has spread to the skin. This is stage 4 cancer, the stage at which most oncologists have ceased trying to stop the cancer.

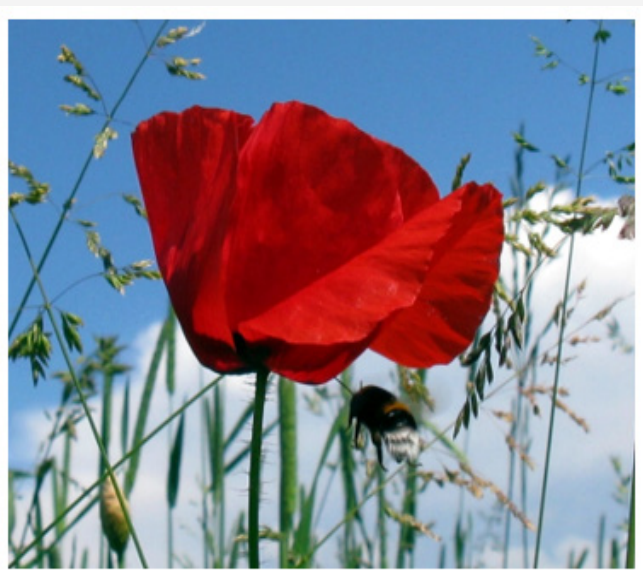

Figure 1: Photo of poppy and bee by the author.

The lady had lost faith in the usual chemo-radiation methods. They didn't work and had cost her a fortune. Word of mouth led her to a CellSonic specialist who was not advertising that CellSonic can treat cancer, but the specialist had previous experience and knew that only CellSonic could help. The doctor asked the lady about the pain on a scale of 1 to 10 . She replied 14. It was agony. He gave her one treatment and the pain went away. It is not clear how quickly the pain was quashed, whether it was immediate or within hours. It was also reported that the scales of scabs started to dry out. The doctor was both pleased and perplexed. The result was better than he expected, and he asked for my opinion. I told him that the result was predictable as we know from cancer cases going back almost three years. There are no side effects. On the third day, the patient feels different. The load on their immune system is greatly reduced and this translates to feeling better.

I said to the doctor that he had shifted from cancer to plastic surgery but to not rush into anything. See if the scabs fall off. Will the tumour, causing the swelling, dissipate or need removing surgically? Now benign, there is every chance that the tumour will be removed by the immune system. He should also be aware that with the pain gone from the breasts, she may become aware of other pains hidden by the predominance of the breast pain. In other words, could the cancer have spread to other organs which have not yet been treated? With such visually obvious damage, there had been no scans. Thus far, there are no further reports of cancer elsewhere.

\section{We await the next report}

Cancer is the replication of mutating cells. Researchers in England have measured the permittivity of tissues and found cancer to show 9.6 and healthy tissue 3.2 on their scale with nothing in between. The CellSonic pulse delivers a high voltage electromagnetic field lasting less than a nanosecond combined with a short duration pressure pulse causing the replication to switch to healthy cells. Laboratory research on cells in Austria four years ago found the same results. No drugs are used and must not be used. The cancer cells should be in good condition so that they respond to the magnetic field. An attempt in Belgium to cure cancer patients failed. The ensuing discussion with the doctor revealed that all the patients were fully loaded with chemotherapy making their cancers unresponsive. He assured me that it would be impossible to find any cancer patients in Europe who were not on chemotherapy.

\section{Non-Cancer Pain}

The traditional view of physiotherapy is that it teaches a few simple exercises and by pressing with fingers some pain can be alleviated. Worse than that, a physiotherapist is said to be inferior to an orthopaedic surgeon. Whether that was ever true, it certainly is not now. Instead of pressing with fingers, the physiotherapist has a new tool in CellSonic that performs non-invasive surgery, does not use drugs and has no side effects. Patients come in bent and walk out straight. Quite apart from the benefits to patients, the status of physiotherapy is enhanced, the business improved, and earnings increased. With their knowledge of the anatomy, all a physiotherapist needs to master the new technology is a few minutes training on how to operate the machine and then they are working inside the body from the outside.

Cellsonic VIPP (very intense pressure pulses) damage or provoke. This is a simplification because the complete explanation is complicated. Damage is done to infection, germs are killed, calcifications shattered, and blockages released. Provocation is the stimulation of the immune system to make a repair by bringing stem cells to the site, increasing vascularization and blood cells. 
Muscles are enhanced and nerves repaired. Additionally, and very simply, the replication of mutant cells is stopped, and they then only replicate healthy cells. In other words, cancer is stopped without drugs, non-invasively and without side effects. It takes a physiotherapist five minutes to realize that they can do more than they were trained to do. Quite apart from curing cancer, which is the easiest treatment to perform, an athlete can be given a $13 \%$ improvement in performance. A geriatric marooned in bed with a catheter and pressure sore can be restored to mobility and dignity. Half the population all of whom have lower back pain can be relieved. What cannot be done easily is to placate orthopods who sense that physios have encroached on their patch.

CellSonic has no apologies. The story of CellSonic is one of discovery. It started with breaking kidney stones with sound waves forty years ago. This is now done in all hospitals of the world and millions of patients have been treated safely. Since then the technology has changed, the machines became smaller, weaker, hand held and cost much less. New applications were reported by users, usually doing something that was not recommended but they thought they would try it. The fact that CellSonic has no side effects made everything possible. This is unique in medicine where all drugs have side effects. Gone is the notion that the body is the sum total of its chemistry. The pharmaceutical industry still has an important role to play but it is no longer the only force in medicine.

\section{Back Pain and Arthritis}

I rely on reports from users of CellSonic for confirmation of the effects of the technology. Here is one from Poland: Below I'm sending a short report from Neuromedyka Clinic in Żyrardów in Poland. Jarosław, 41 years old patient with a heel spur. Mr. Jarek had a very big problem with his leg. He felt a strong pain even he doesn't walk. We did 3 treatments to solve his problem. It took us 4 weeks. After 1st treatment he doesn't feel significant improvement, but he decided to take all treatments. After 2nd treatment he felt much better only pain appears after all day when he walked. I called him today, it is 4 weeks after last treatment and asked him how he is feeling? He said that he doesn't remember that he had any problems with his leg. He feels great.

These photos are from Latin America (Figure 2):

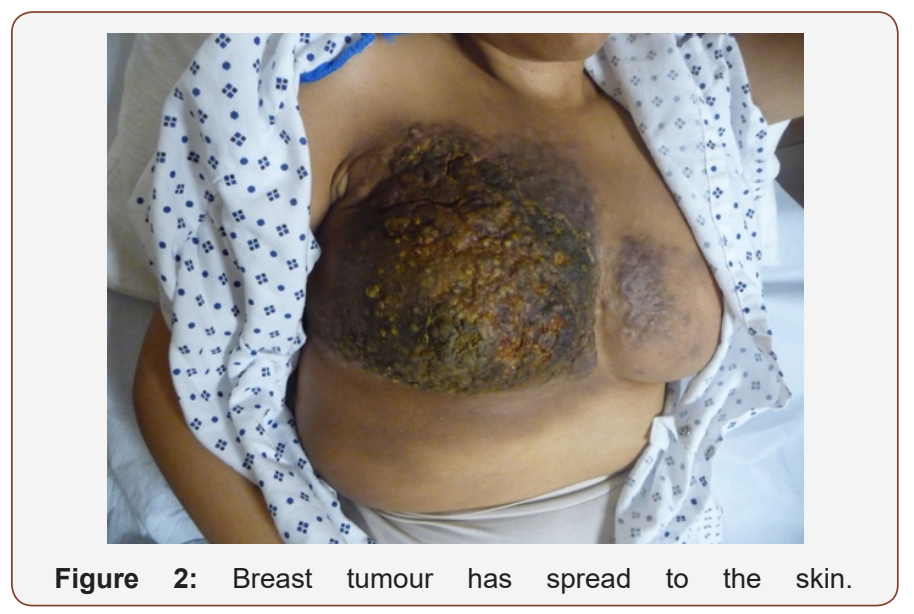

The lady could not lift her arm. Fifteen minutes after a fiveminute treatment with CellSonic she could.

In $80 \%$ of cases, tennis elbow is cured with one CellSonic treatment of five minutes duration (Figure 3).

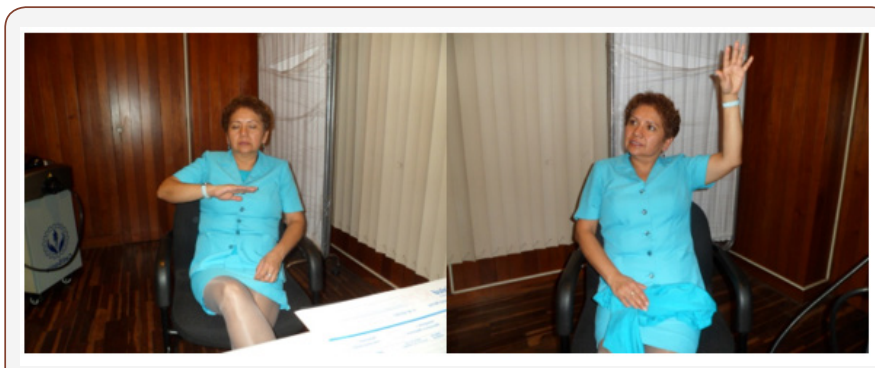

Figure 3: The lady could not lift her arm. Fifteen minutes after a five-minute treatment with CellSonic she could.

\section{Vipp for Treatment of Calcaneal Spur and Fasciitis}

The next report is from India. Bony spurs on the heel of foot may be a cause of pain in individuals, which may increase in intensity after prolonged periods of rest. Walking, running or lifting heavy weights may exacerbate the condition. These spurs are a result of repeated stress due to which calcium deposition occurs. Similarly, plantar fasciitis is a condition that occurs in individuals, commonly due to prolonged standing. The condition is due to inflammation at the site of insertion of ligaments into the bone. Pathologically, micro tears, collagen breakdown and scarring are observed. Pain is the main feature of plantar fasciitis, which is usually most severe on taking the first step after prolonged periods of rest. Both conditions, commonly being associated with constant stress on legs and feet, treatment comprises of pain-relieving medications, lifestyle modifications and physiotherapy exercises.

A new treatment modality utilizing CellSonic VIPP technology has shown improvement in both conditions, as seen in patients treated at StemRx Bioscience Solutions Pvt. Ltd. 5 patients with spur/fasciitis were given a total of 3 sessions of VIPP treatment. First and second sessions were given at an interval of 3 days, while the 3rd session was given a week following 2 nd session. 200500 shocks were given at intensity level 2-4. Pain relief was the immediate effect noticed. At 3-4 weeks follow-up, all patients were pain free and had improved comfort in walking ability. All patients have stopped pain medications. "It is amazing to see positive results in a short period of time. Being a non-invasive, drug and side effect free procedure with no hospitalization requirement, CellSonic VIPP is a hit among patients with calcaneal spur/fasciitis", says Dr. Mahajan.

In all the cases, drugs are not used, nerves are repaired, vascularization improved to carry oxygen and stem cells of the right type to the right place in the right quantity and infection killed without anti-biotics (Figure 4). Lower back pain could be the predominant pain world-wide (Figure 5). Usually one CellSonic treatment is enough for permanent relief. The ultimate test on spine repair is a severed spinal cord. I am now aware of three cases, one in Hyderabad on a girl whom I met so I can vouch for the authenticity 
and two cases from Dr Mahajan in Mumbai whom I know well and have watched him treating patients.

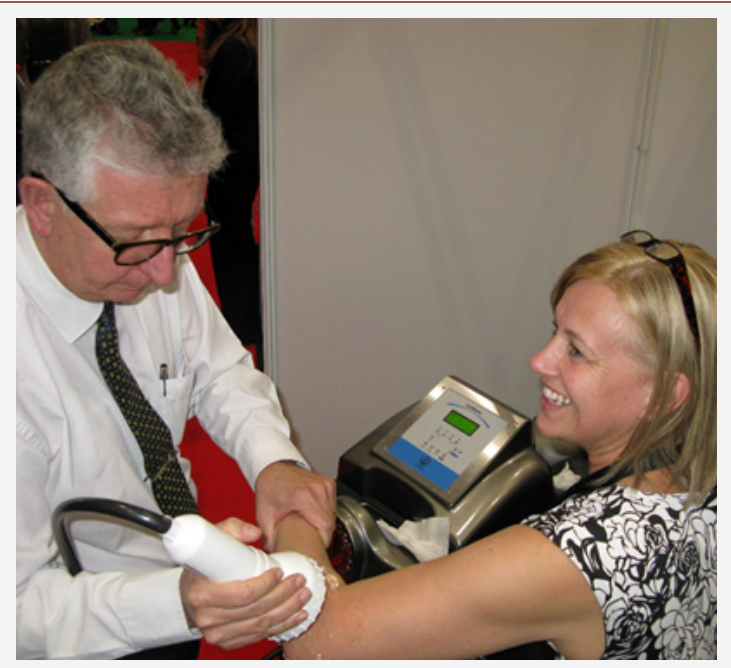

Figure 4: In $80 \%$ of cases, tennis elbow is cured with one CellSonic treatment of five minutes duration.

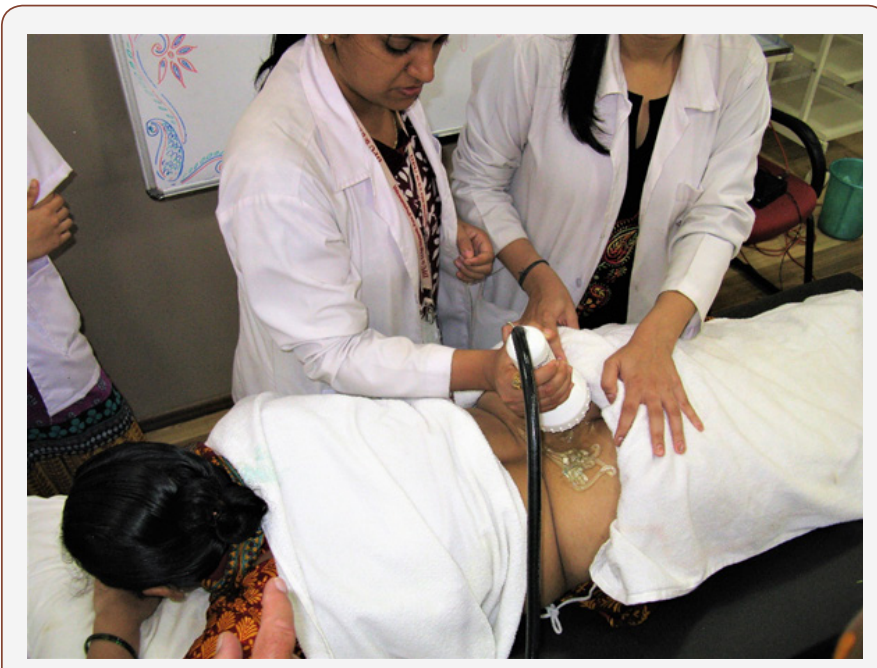

Figure 5: Lower back pain could be the predominant pain worldwide. Usually one CellSonic treatment is sufficient for permanent relief.

\section{Spinal Cord Injury Patient Treated with Cell-Based} Therapy and Cellsonic VIPP

The patient had a road traffic accident and sustained injuries in his spine in September 2017. He was paralyzed below the waist and was bedridden since the accident. He did not undergo any major treatment at his home country. He was brought to our hospital on a stretcher. With cell-based therapy and CellSonic VIPP plus neurorehabilitation, the patient was able to stand with the help of callipers within a month of treatment. This is tremendous improvement as the family was told that the boy will remain bedridden. He had developed bed sores due to lack of movement. With the combination treatment of cell-based therapy and CellSonic VIPP in the wound area, we could accomplish rapid healing of the sores. Overall the improvement is very encouraging. After few months of follow up, we will look to publish this case. We have few more cases in the pipeline, and we hope to publish them in reputed journals after the required follow up period.

\section{Emotional Pain}

Physical pain causes emotional pain. It is a downward spiral. The body is healed by the immune system which depends on a positive frame of mind [11]. The expression, Mind over Body, cannot always apply. I have heard it used with reference to cancer so it may be possible to think yourself well but for the general population it is not an easy remedy. People have emotional states that range from fragile to robust. For some, a day of sky covered cloud with no sign of the sun brings on doom. For others, they can emerge from battle in a war zone and continue their lives as though nothing dramatic has happened. Somewhere between these two extremes are most of us. The trick is to find what makes you smile. Not to laugh or be giddy. Just something that is pleasing, and you find yourself saying, "If it could always be like this!" You then have something to aim for because those pleasant times are repeatable.

I cannot think of anyone I have known who has been on drugs (anti-depressants) to shift them away from the gloom end of the spectrum who has been able to permanently achieve equilibrium. If at first the drugs improved the patient's outlook, before long they were damaging the brain's emotional balance. The factors involved are the amount of the dose and its duration. When a patient says they are on a low dose and believing it is not causing dependency they are not realizing that every day a piece of their brain becomes permanently inactive. The damage is accumulative. The drug may help them to struggle on, but it is preventing a cure.

The human brain evolved to cope with life's swings. Our emotions exist for a reason. Combined with memory, they are a protective mechanism. I have often been tempted to write an article entitled, "Blame the brain" and when I assemble my thoughts, I found I was entering taboo areas such as religion and politics. My purpose is to help, and the measure of success is more people recovering. If my conjecture is taken to be critical of some beliefs, then I cannot help and will avoid the subject. You must work out for yourself what avoids friction.

We are gregarious. Who would choose to live alone? Has a hermit an enquiring mind? Do they ever smile? Those around us depend on us as we depend on them. Be amenable. Within a range of moods, all people are the same and I say that knowing people from all around the world. Only the ignorant can be xenophobic.

You must exercise [12], eat the right food [13] and never smoke, drink alcohol or take narcotics. If you damage the brain, all is lost [14]. When people continue to use opioids beyond what a doctor prescribes, whether to minimize pain or induce euphoric feelings, it can mark the beginning stages of an opiate addiction, with a tolerance developing and eventually leading to dependence, when a person relies on the drug to prevent withdrawal symptoms [9]. Writers have pointed to a widespread desire among the public to find a pill for any problem, even if a better solution might be a lifestyle change, such as exercise, improved diet, and stress reduction [10-12]. Opioids are relatively inexpensive, and alternative interventions, such as physical therapy, may not be affordable [13]. 
I have put the key words of the above quote in bold: a pill for any problem. And that is the problem. Medical practice has created the problem. From the Opium wars inflicted on China in the 19th Century to the present-day Opioid Crisis, the people suffer because they allow themselves to be weak and ignorant. Doctors who take a stance can be ridiculed and even banned from practicing.

\section{Causing Happiness}

CellSonic has been used on many people for enough years to observe a phenomenon I do not yet understand, it makes them happy. As reports flowed back to me, they almost always said that the patient enjoyed the treatments, looked forward to another treatment and the spouse also would add that the patient felt happier. It assumed this was the charm of the doctor and almost painless, quick treatment. Eventually, as the stories accumulated, I had to accept that there was more to it than a smiling doctor. The effect was most noticeable where many shocks were applied and had to be repeated every few days on such as gangrene, kidney failure or the severed spinal cord. CellSonic pulses were making the patient feel happier.

I do not know whether we have a non-pharmaceutical cure for depression. At some stage it will be worth gathering some depressives who are not on medication and treating them. The hypothesis is that it does to the brain what exercise does and, importantly, carries nothing into the brain that can destroy connections or cause blockages. This is the next frontier or at least one of them. Other projects for CellSonic are diagnosing cancer harmlessly.

\section{Conclusion}

In most cases, pain can be removed by healing the cause of the pain with CellSonic and it can be done without side effects and without brain bending drugs. Moreover, the cost of CellSonic treatments is less than other methods which are ineffective. The benefits flow to the people, their costs of living and their governments. This technological change is disruptive and like all such changes in history the adjustment may not be smooth, but it is inevitable. CellSonic gives people what they want. What's better than a cure in a pill? Answer, a safe cure not in a pill.

\section{Acknowledgement}

None.

\section{Conflict of Interest}

No conflict of interest.

\section{References}

1. http://www.bbc.com/future/story/20140528-do-animals-take-drugs. https://news.nationalgeographic.com/2015/11/151121-animalsscience-drunk-insects-mammals-drinking.

2. https://en.wikipedia.org/wiki/Opium/https://www.chemistryworld. com/podcasts/morphine/3005864.article

3. https://michaelpollan.com/articles-archive/opium-made-easy.

4. https://www.nytimes.com/2018/07/03/world/asia/opium-warbook-china-britain.html/ https://www.historytoday.com/julia-lovell/ opium-wars-both-sides-now/https://www.nam.ac.uk/explore/opiumwar-1839-1842.

5. https://en.wikipedia.org/wiki/Opioid_epidemic.

6. https://www.poison.org/articles/opioid-epidemic-history-andprescribing-patterns-182.

7. https://www.drugabuse.gov/drugs-abuse/opioids/opioid-overdosecrisis.

8. https://www.psychologytoday.com/gb/blog/nation-in-pain/201511/ chronic-pain-and-the-risk-suicide.

9. http://www.lynnwebstermd.com/suicide-and-chronic-pain.

10. How the cure for cancer was discovered, Cancer Cure-the protocol and explanation, Cancer's change of direction, Professor Hague's Discovery, Review of reports of curing cancer with CellSonic VIPP machines, Inflammatory Breast Cancer, Leprosy and Cancer.

\section{CellSonic VIPP Naturally.}

12. The Exercise Continuum.

13. The ins and outs of elimination.

14. https://en.wikipedia.org/wiki/Opioid_epidemic. 